\title{
Philosophiques
}

Niklas Luhmann, Amour comme passion. De la codification de l'intimité, traduit de l'allemand par Anne-Marie Lionnet, Aubier, 1990, (c 1986), 330 p.

Niklas Luhmann, Ecological Communication, Translated by John Bednarz Jr., The University of Chicago Press, 1989, (1986), $187 \mathrm{p}$.

\section{Guy Bellavance}

Volume 18, numéro 2, automne 1991

URI : https://id.erudit.org/iderudit/027159ar

DOI : https://doi.org/10.7202/027159ar

Aller au sommaire du numéro

Éditeur(s)

Société de philosophie du Québec

ISSN

0316-2923 (imprimé)

1492-1391 (numérique)

Découvrir la revue

Citer ce compte rendu

Bellavance, G. (1991). Compte rendu de [Niklas Luhmann, Amour comme passion. De la codification de l'intimité, traduit de l'allemand par Anne-Marie Lionnet, Aubier, 1990, (c 1986), 330 p. / Niklas Luhmann, Ecological

Communication, Translated by John Bednarz Jr., The University of Chicago

Press, 1989, (1986), 187 p.] Philosophiques, 18(2), 177-180.

https://doi.org/10.7202/027159ar d'utilisation que vous pouvez consulter en ligne. 


\section{COMPTES RENDUS}

Niklas LUHMANN, Amour comme passion. De la codification de l'intimité, traduit de l'allemand par Anne-Marie Lionnet, Aubier, 1990, (c 1982), 300 p.

Niklas LUHMANN, Ecological Communication, Translated by John Bednarz Jr., The University of Chicago Press, 1989, (c 1986), 187 p.

par Guy Bellavance

Ces deux titres s'ajoutent au corpus encore parcimonieux des traductions d'une des plus stimulantes contributions à la théorie sociologique contemporaine. Théoricien érudit et prolifique (plus de 6000 pages de textes en édition allemande), original et controversé, la plupart du public francophone a sans doute été introduit à ses positions par le truchement de la critique qu'en fait Jürgen Habermas (cf. Le discours philosophique de la modernité), dont Luhmann est en Allemagne le principal contradicteur. Ceci rend d'autant plus problématique la stratégie de traduction adoptée ici. Amour comme Passion, sans être un ouvrage mineur, manque l'arrière plan théorique indispensable pour en apprécier - notion chère à Luhmann - toute la " complexité ». A cet égard, Ecological communication, qui offre une plus large vue de l'ensemble théorique, qui couvre un plus grand nombre de domaines sociaux et qui expose des thèses souvent plus provocantes, est plus satisfaisant. Le francophone anglophile peut aussi se référer à deux autres ouvrages, The Differentiation of Society (1982) et Trust and Power (1979), où se trouvent les mêmes vues générales et qui comportent de surcroît des introductions fouillées. En français on devra pour l'instant se contenter de trois articles dispersés dans autant de revues différentes: "L'invraisemblance de la communication " Reoue Internationale de Science Sociale, 1981, "Remarques préliminaires en vue d'une théorie des systèmes sociaux » Critique (trad. C. Piché), 1981 et "L'État politique » Traverses, 1985.

L'intérêt, et la difficulté, du programme théorique de Luhman tient à son développement au confluent de plusieurs traditions intellectuelles: systémisme fonctionnaliste (à la suite de Parsons), cybernétique, développements épistémologiques de la biologie et phénoménologie. Cet effort de synthèse vise à intégrer à l'analyse de la société, et principalement des sociétés contemporaines, plusieurs champs théoriques: théorie de la communication, théorie des systèmes et théorie de 
l'évolution socioculturelle. Chacun de ces axes théoriques s'articule autour de thèmes principaux qui permettent de situer rapidement, et sans doute trop grossièrement, l'auteur: invraisemblance de la communication et de l'interaction sociale, marquées par la double contingence; instabilité intrinsèque des systèmes sociaux, définis à titre d'actes communicationnels, et rivalisant en complexité avec les turbulences d'environnements qui leurs servent à la fois d'horizons et de prémisses; évolution d'une forme de société stratifiée (dont l'unité est assurée par un centre ou le sommet d'une hiérarchie) à une forme de société acentrée soumise au principe de différenciation fonctionnelle des sous-systèmes, chacun d'eux comme autant de points d'expression de l'unité de l'ensemble du système. Comme le dit l'auteur, l'unité de la société n'est rien de plus que la différence des systèmes fonctionnels (E.C. p.114). Chacun de ses ouvrages est ainsi conçu en fonction de ces différents niveaux conceptuels et déployé en référence à ces multiples traditions intellectuelles. En ce sens, Ecological Communication, critique des récents mouvements écologiques et des littératures qui en ressortent, est d'abord l'occasion d'expliquer les contraintes particulières que fait peser la différenciation fonctionnelle sur les relations entre système social et environnement, et, du même coup, une tentative de situer le problème écologique, tel qu'il s'est construit, en fonction de cette contrainte propre à la société moderne. Par là, le sujet de cet ouvrage, la "communication écologique", n'est pas le danger écologique en tant que tel, mais la forme obligée que doit suivre le processus de communication qui conduit à être averti de l'existence d'un tel danger. Ici, Luhmann fait intervenir la notion de "résonance " (processus d'ajustement continu de la société aux changements environnementaux) pour souligner l'état de communication décalée qu'entretient le système à son environnement (ceci à l'encontre des théories des écosystèmes). Il montre aussi comment la différenciation systémique, amplifiant l'indétermination du système social, contribue à accroître le niveau de communication écologique, forçant de surcroît à une posture paradoxale d'autoobservation sociale. La thèse principale, à savoir que les changements environnementaux produisent à la fois trop et trop peu de résonances au sein de la société, tient à cette particularité des systèmes d'être à la fois ouverts de l'intérieur et fermés sur eux-mêmes, obligeant à distinguer environnements interne et externe: au plan des frontières externes, les résonances peuvent être trop faibles, à cause du principe de restriction autour duquel s'organise tout système autoréférentiel; au plan des frontières internes elles seront trop fortes, compte tenu des niveaux de performance accrus de chacun des différents soussystèmes, de leur extrême sensibilité, amplifiant d'autant risques d'interférences et problèmes de coordination. Luhmann accorde dès lors beaucoup d'attention aux contraintes propres à chacun des principaux sous-systèmes sociaux - droit, économie, politique, science, religion, éducation - à leurs codes et programmes respectifs, et 
aux interférences et perturbations communicationnelles des uns aux autres. Ceci l'amène à une critique du zèle moral des nouveaux mouvements sociaux, substitut à un déficit théorique quant aux contraintes de la différenciation fonctionnelle, et à d'importantes réserves adressées à tout projet d'éthique écologique, de même qu'à une analyse originale de la fonction (rhétorique) de l'anxiété, thème porteur des nouvelles revendications et, selon Luhman, seuil minimal de communication écologique.

Par contraste, Amour comme passion, représente une démarche diachronique, appuyée sur un vaste corpus littéraire (du XVle siècle à aujourd'hui) et centrée sur l'évolution d'un code tout particulier, et de sa sémantique. C'est de plus l'occasion de mettre en oeuvre une notion cardinale à sa théorie de la communication, celle de "médium généralisé de communication symbolique ". L'Amour, désigné comme ce médium auquel a été assigné la tâche d'assurer le traitement communicationnel de l'individualité, est ainsi traité "comme code symbolique qui informe sur la manière dont on peut, dans le cas où cette éventualité est plutôt invraisemblable, communiquer tout de même avec succès » (p.18). Cherchant comment le médium a pu évoluer à travers ce passage d'une forme de société traditionnelle (organisée par stratification) à la forme contemporaine de différenciation, l'auteur résume cette évolution par la formule d'un " accroissement de vraisemblance de l'invraisemblable" (Ibid.). L'Amour, tentative de réponse à la dynamique de dissolution inhérente à toute liaison, enregistre aussi à travers sa sémantique, à titre de Médium, le processus de normalisation de structures sociales plus invraisemblables. Examinant le déplacement du centre de gravité du médium - qui passe de l'idéal (classique), au paradoxe (romantique) avant de devenir problème (fonctionnel) - il en profite pour mettre en lumière les contraintes liées au principe de "double contingence". L'ouvrage s'achève sur une analyse de la problématisation contemporaine du code, où la différence entre relations personnelles et impersonnelles tend à devenir la "différence ", celle "qui fait la différence". L'amour autonome, confondu à la recherche de l'autonomie personnelle, est alors recherché à titre de "validation de la présentation de soi ", chacun laissé à lui-même face à l'autre aux prises avec un problème de communication improbable. La société se trouve en ce sens confrontée aujourd'hui à ses désirs sous forme de réalité. L'Amour, "comme problème du maintien de l'invraisemblance, est rendu conscient " (p. 210), imposant un niveau d'exigences toujours plus élevé, producteurs d'attentes d'autant plus souvent déçues. L'Amour Passion apparaît dès lors sémantique de transition, ayant permis le développement réflexif de l'intimité (à base sexuelle), ayant aidé à promouvoir puis à imposer l'Amour, mais semblant plutôt inapte à traiter ce qui en est résulté: "Rendre d'abord possible, et aussi plausible, ce qui est 
invraisemblable est une chose; mais le supporter en est une autre " (p. 199).

Département de Sociologie

Université de Montréal

S 